\title{
Robotic Monitoring of Gravitationally Lensed Quasars
}

\author{
Luis J. Goicoechea, ${ }^{1}$ Vyacheslav N. Shalyapin, ${ }^{2}$ and Aurora Ullán ${ }^{3}$ \\ ${ }^{1}$ Departamento de Física Moderna, Universidad de Cantabria, Avda. de Los Castros s/n, 39005 Santander, Spain \\ ${ }^{2}$ Institute for Radiophysics and Electronics, National Academy of Sciences of Ukraine, 12 Proskura Street, Kharkov 61085, Ukraine \\ ${ }^{3}$ Robotic Telescopes Group, Centro de Astrobiología (CSIC-INTA), Ctra de Ajalvir, km 4, Torrejón de Ardoz, 28850 Madrid, Spain \\ Correspondence should be addressed to Luis J. Goicoechea, luis.goicoechea@unican.es
}

Received 22 June 2009; Accepted 23 November 2009

Academic Editor: Joshua S. Bloom

Copyright ( $\odot 2010$ Luis J. Goicoechea et al. This is an open access article distributed under the Creative Commons Attribution License, which permits unrestricted use, distribution, and reproduction in any medium, provided the original work is properly cited.

\begin{abstract}
We report on the first observational phase of the Liverpool Quasar Lens Monitoring (LQLM) project. This mainly consisted of the optical follow-up of three lensed quasars using the $2 \mathrm{~m}$ Liverpool Robotic Telescope. The observational subprogram started in January 2005 and was completed in July 2007. We also describe our photometric approaches (including two pipelines to extract accurate and reliable fluxes of images of lensed quasars), the performance of the telescope when taking modest nightly exposures of lens systems, and the main scientific results from the observed light curves. The LQLM archive and the current status of the project (second phase) are also outlined.
\end{abstract}

\section{Introduction}

If there is a massive galaxy (or an association of galaxies) between a background quasar (QSO) and the observer, the QSO is seen as a multiple system consisting of several images A, B, C,... (see, e.g., [1]). In the simplest case, the gravitational deflector produces two bright images (A and B) of the same distant QSO. As the ray paths are different for the two images of the double QSO, the corresponding magnifications and travel times do not agree with each other. Thus, each flare in the distant source is observed in A and B with different flux peak (magnitude offset) and arrival time (time delay). Time delays of gravitationally lensed QSOs are basically related to the present expansion rate of the Universe (Hubble constant) and mean surface densities of main lensing galaxies $[2,3]$ as well as flaring regions where the luminosity fluctuations occur $[4,5]$. Moreover, delays between intrinsic variations observed at different wavelengths could unveil the origin of QSO flares. Structure function analyses also allow one to quantify typical luminosity fluctuations at different rest-frame lags. Hence, these analyses may reveal important aspects of the mechanism of intrinsic variability in QSOs [6, 7]. Apart from intrinsic variations, extrinsic signals are detected in some lensed QSOs (see, e.g., [8]). These extrinsic events are very probably due to small-scale substructures in main lensing galaxies, that is, stars and other collapsed objects (gravitational microlensing), or clouds of dust (variable transmission). The study of extrinsic variability has a great interest, since one can obtain information about the nature of sources (QSOs), as well as the composition of intervening galaxies (see, e.g., [9-11]).

Accurate measurements of time delays (ranging from days to years) obtained from optical intrinsic events (timescales of weeks-months) require intensive long-term monitoring campaigns, that is, nightly observations over relatively long periods. An intensive long-term sampling is also required for detailed studies of structure functions or extrinsic variations. Moreover, bright-lensed QSOs have image separations and visual fluxes of $\Delta \theta \sim 1-10^{\prime \prime}$ and $m_{V} \sim$ 17-20 mag [12]. Taking these values into account, one also needs high-performance telescopes (good angular resolutions, relatively large primary mirrors, reliable instruments, etc.). Unfortunately, space and largest ground-based telescopes are not available for observational programs involving such exigent samplings. Only some $1-3 \mathrm{~m}$ conventional telescopes and the $2 \mathrm{~m}$ Liverpool Robotic Telescope (LRT) are being used for systematic monitoring of lensed QSOs. 
TABLE 1: LQLM I optical frames.

\begin{tabular}{lccccc}
\hline Target & $z$ & $\Delta \theta_{\mathrm{AB}}\left(^{\prime \prime}\right)$ & $\begin{array}{c}g \text {-band } \\
\text { frames }\end{array}$ & $\begin{array}{c}r \text {-band } \\
\text { frames }\end{array}$ & $\begin{array}{c}i \text {-band } \\
\text { frames }\end{array}$ \\
\hline $\begin{array}{l}\text { SBS } \\
0909+532\end{array}$ & 1.38 & 1.1 & 237 & 214 & - \\
$\begin{array}{l}\text { QSO } \\
0957+561\end{array}$ & 1.41 & 6.2 & 286 & 264 & - \\
$\begin{array}{l}\text { FBQ } \\
0951+2635\end{array}$ & 1.24 & 1.1 & - & - & 259 \\
\hline
\end{tabular}

The LRT [13, 14] at the Roque de los Muchachos Observatory (Canary Islands, Spain) is one of the world's largest fully robotic telescopes, and it is ideally suited for monitoring lens systems and for deriving light curves of their components. In this paper we review the Liverpool Quasar Lens Monitoring (LQLM) project, which is being conducted by us. In Section 2, we give a brief description of the phase I (LQLM I) observations. In Section 3, we discuss the photometric methods and focus on two pipelines that are specially designed for the analysis of large sets of frames of lensed QSOs. In Section 4, we give an overview of the observed light curves and the behaviour of the telescope (effective sampling and photometric accuracy). In Section 5, we summarize the main scientific outputs of the LQLM I subproject. In Section 6, we present the LQLM archive and the current status of the project (phase II).

\section{LQLM I Observations}

The first phase of the nightly long-term observational program with the LRT began in January 2005 and finished in July 2007. This LQLM I subprogram was carried out with the RATCam optical CCD camera on the LRT, using gri Sloan filters. The field of view and the pixel scale (binning $2 \times 2$ ) were $\sim 4.6^{\prime} \times 4.6^{\prime}$ and $0.278^{\prime \prime}$, respectively. The subprogram mainly focused on the optical follow-up of three bright double QSOs at $1 \leq z \leq 2$ : SBS $0909+532$, QSO $0957+561$ and FBQ $0951+2635$ (see [12] for HST frames, basic properties, and references). During our 2.5year monitoring campaign, we obtained 1260 individual frames with exposure times ranging from 100 to 200 seconds. The distribution of frames is shown in Table 1.

While SBS $0909+532$ and QSO 0957 + 561 were monitored in the $g$ and $r$ Sloan bands over two years (until January 2007) and the whole 2.5-year period, respectively, the monitoring campaign of FBQ $0951+2635$ in the $i$ Sloan band only lasted four months (in 2007). The longterm follow-up of SBS $0909+532$ and QSO $0957+561$ is characterised by two important seasonal gaps. These are a consequence of the simultaneous annual occultation of both lens systems. For SBS $0909+532$ and QSO $0957+561$, in most observation nights we got one exposure in each filter (sometimes two or three exposures were taken with the LRT). With respect to FBQ $0951+2635$, each observation night consisted of five exposures of 100 seconds in the $i$ band, using a dither cross pattern.

\section{Photometric Approaches and Pipelines}

The LRT preprocessing pipeline included bias subtraction, trimming of the overscan regions, and flat-fielding. We also performed other basic instrumental reductions: cosmic rays cleaning, bad pixel correction, and defringing ( $i$ band frames). The next step was a preselection, based on individual inspection of frames and headers. We initially removed all frames that either do not include the lens systems or are characterised by an anomalous image formation or have large seeing discs (FWHMs). For example, only QSO $0957+561$ frames verifying FWHM $<3^{\prime \prime}$ and FBQ 0951 +2635 frames with FWHM $\leq 2^{\prime \prime}$ were considered for subsequent analyses. Note that our FWHM limits are not consistent with each other, since the constraint on QSO $0957+561\left(\mathrm{FWHM}<\Delta \theta_{\mathrm{AB}} / 2\right)$ is consistent with an upper limit of $0.5^{\prime \prime}$ for the compact systems (see the corresponding angular separations in Table 1). Unfortunately, at present there are no available facilities to carry out intensive longterm monitoring projects of compact-lensed QSOs under such extremely good seeing conditions. For FBQ 0951 +2635 , all preselected frames in each night $(\leq 5)$ were combined; that is, they were aligned and then averaged.

SBS $0909+532$ consists of two images of the same distant QSO $(z=1.38)$, which are separated by about $1^{\prime \prime}$ (see Table 1). The lensing galaxy is not apparent in optical frames, even using the HST [12]. Thus, a simple photometric model (with only two close stellar-like sources, i.e., two empirical PSFs) is able to describe the whole crowded region associated with the QSO images. Our second target was the first gravitationally lensed QSO: QSO 0957 + 561 or Twin QSO. It also consists of two bright images (A and B) of a QSO at $z \sim 1.4$. However, in this case, the separation between both QSO images is of about 6", and there is a relatively bright lensing galaxy near to B ( 1 " apart). This giant elliptical galaxy is clearly detected in HST optical frames [12]; so it is taken into account when modelling the system. The photometric model incorporated two empirical PSFs (to fit both QSO images) and a de Vaucouleurs profile convolved with the empirical PSF (to fit the lensing elliptical galaxy). The third target FBQ $0951+2635$ is compact (like SBS $0909+532$ ) and includes a relatively bright lens galaxy very near to the B QSO image (only 0.2" apart). It is not possible (or very difficult) to separate the galaxy signal from the B signal and to resolve the system into three individual contributions under normal seeing conditions, that is, FWHM $=1-2 "$. Despite efforts to quantify the galaxy signal in LQLM I frames, we inferred meaningless results. Therefore, we adopted a simple photometric model (two empirical PSFs), where the B flux represents the true flux of $\mathrm{B}$ plus some contamination by galaxy light.

For a given target, once the most suitable photometric model (including associated procedures and constraints) is decided, the corresponding crowded-field photometry pipeline (CPP) does photometry of bright field stars and QSO images. This pipeline is written in Python programming language and incorporates the capabilities of IRAF, through the PyRAF interface, and IMFITFITS software [15], as well as additional numerical and graphical tools. 


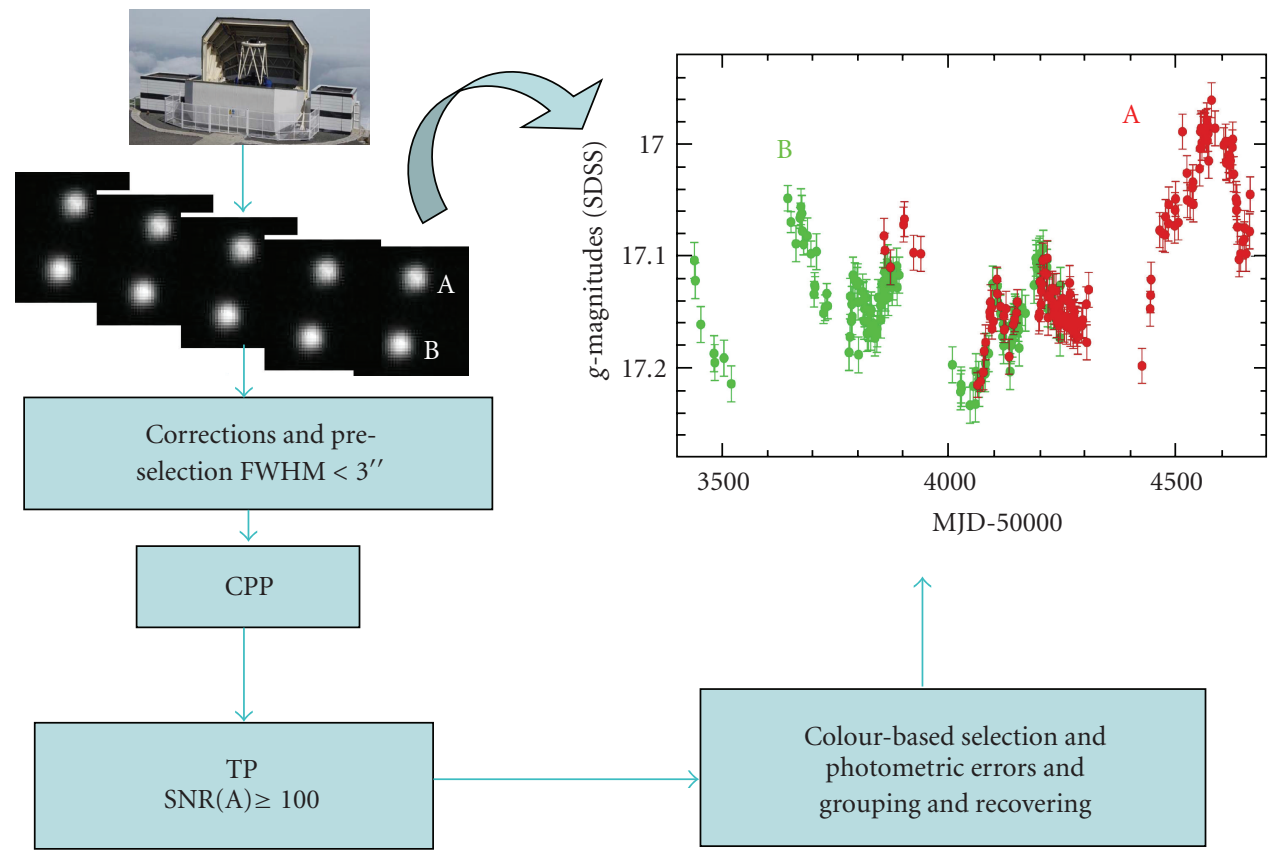

FIGURE 1: Photometric scheme for QSO $0957+561$ : from LRT frames in the $g$ band to SDSS magnitudes of both images (see main text for details).

The IMFITFITS software has been developed to analyse gravitational lens systems. We also used a transformation pipeline (TP; in Python programming language) to obtain SDSS magnitudes from instrumental magnitudes that are corrected for systematic effects. The transformation model incorporates zero-point, colour, and inhomogeneity corrections.

For example, the photometric scheme for QSO $0957+$ 561 in the $g$ band is outlined in Figure 1 (see also [16]). The TP was initially applied to frames with signal-to-noise ratio $(\mathrm{SNR}) \geq 100$ over the A image (both images have similar brightness). This automatic task fits the deviations between instrumental and standard (SDSS catalogue) magnitudes of several reference stars to the transformation model. The zero-point term and the colour coefficient are allowed to vary over time, and the inhomogeneity term contains linear and quadratic coefficients (see, e.g., [17, 18]). From the TP output, we derived two relevant results: (a) there are some dates in which the colour coefficient very strongly deviates $(\sim 100 \%)$ from its average value, and $(b)$ the $2 \mathrm{D}$ inhomogeneity pattern has an amplitude of about $80 \mathrm{mmag}$, and so the inhomogeneity correction plays an important role in getting accurate fluxes. The extreme deviations in the colour coefficient were very probably related to atmosphericinstrumental problems, and so the associated frames were removed from the dataset (colour-based selection). Sometimes an atmospheric-instrumental perturbation produces an anomalous behaviour of the colour coefficient that is associated with a systematic artifact in the fluxes of all images of a lensed QSO. Thus, we must be careful when interpreting the origin of short-timescale variations in lensed QSOs.
We used the standard deviation between adjacent magnitudes (time separation $\leq 3$ days) as an error estimator. This works with stellar records, and moreover, QSO $0957+$ 561 is not an optically violently variable QSO. We obtained self-consistent uncertainties for stars and QSO images. The final step consisted of grouping pairs or trios of magnitudes measured on the same night. These final light curves in the $g$ band are incorporated in our current LQLM I data-tools release. However, during the analysis of the time delays of the Twin QSO, we recovered some critical frames $(80<\mathrm{SNR}(\mathrm{A})$ $<100)$ to improve the information on the time evolution of $A$ [16]. The A light curve in Figure 1 includes these additional fluxes. In Figure 1, we also shift the A record by +417 days (time delay) and $-0.089 \mathrm{mag}$ (magnitude offset) to better assess the origin of the observed variability.

\section{LQLM I Light Curves and Performance of the LRT}

Several LQLM I light curves have been presented in recent months $[7,16,19]$. For example, the $r$-band records of SBS $0909+532$ from January 2005 to June 2006 are based on 153 original frames. However, a useful database containing 92 frames (after corrections and preselection) was used to make the light curves [7]. Hence, the success rate (useful frames/original frames) was $60 \%$, which is really impressive if one rethinks the observational procedure, that is, photometric exposures taken in a fully automatic (robotic) way; so one cannot correct the telescope pointing (when it fails), adapt the exposure time to the atmospheric-moonlight conditions, and so forth. This means an effective sampling 
TABLE 2: Performance of the LRT when taking modest nightly exposures of double QSOs with images A and B.

\begin{tabular}{lccccc}
\hline Target/filter & $\begin{array}{c}\text { Sampling } \\
\text { period } \\
\text { (days) }\end{array}$ & $\begin{array}{c}\sigma\left(m_{\mathrm{A}}\right) \\
(\mathrm{mmag})\end{array}$ & $\begin{array}{c}\varepsilon_{\mathrm{A}} \\
(\%)\end{array}$ & $\begin{array}{c}\sigma\left(m_{\mathrm{B}}\right) \\
(\mathrm{mmag})\end{array}$ & $\begin{array}{c}\varepsilon_{\mathrm{B}} \\
(\%)\end{array}$ \\
\hline SBS 0909+532/r & 4 & 14 & 1.3 & 18 & 1.7 \\
QSO 0957+561/g & 3.7 & 16 & 1.5 & 16 & 1.5 \\
QSO 0957+ 561/r & 4.3 & 12 & 1.1 & 12 & 1.1 \\
FBQ 0951 + 2635/i & $5-6$ & 14 & 1.3 & - & - \\
\hline
\end{tabular}

period (excluding the $\sim 120$-day seasonal gap) of about 4 days, that is, $\sim 2$ useful 120 seconds exposures per week (see Table 2). Although pipelines and other posterior refinements were not considered in this first part of the project, we checked the time evolution of the QSO images by means of a parallel $R$-band monitoring at the Maidanak Observatory. The photometric uncertainties were $\sigma\left(m_{\mathrm{A}}\right) \sim 14 \mathrm{mmag}$ $\left(m_{\mathrm{A}} \sim 16.4 \mathrm{mag}\right)$ and $\sigma\left(m_{\mathrm{B}}\right) \sim 18 \mathrm{mmag}\left(m_{\mathrm{B}} \sim 17 \mathrm{mag}\right)$, and we unambiguously detected $50-100 \mathrm{mmag}$ intrinsic variations. The corresponding photometric accuracies were $\varepsilon_{\mathrm{A}} \sim 1.3 \%$ and $\varepsilon_{\mathrm{B}} \sim 1.7 \%(\varepsilon=\sigma(F) / F=0.921 \sigma(m)$, where $F$ is the physical flux). In Figure 2, we show SDSS magnitudes of the A image in the 2005-2006 season (from October 2005 to June 2006). The $r$-band record (red triangles) traces a 50 mmag intrinsic gradient around day 3800 . This is also detected in the $g$ band record (green circles) of the brightest image A. To properly compare the behaviours in both bands, the bluer ( $g$-band) record is shifted by $-0.57 \mathrm{mag}$.

All LQLM I frames of QSO 0957 + 561 were fully analysed using the photometric scheme in Figure 1. After corrections and preselection (FWHM $\left.<3^{\prime \prime}\right)$, the global database contained 199 frames in the $g$ band and 210 frames in the $r$ band. This means that $\sim 75 \%$ of the original LRT frames were selected as input for the CPP (see previous section). Before applying the TP, we removed the frames with $\operatorname{SNR}(\mathrm{A})<100$. The database was also rechecked for anomalies in the image formation, which led to the rejection of the $r$-band exposures taken during the first semester of 2005. The initial high-quality database consisted of $170 \mathrm{~g}$ band and $167 r$-band frames (TP input), that is, about $60 \%$ of the original ones. We then found some anomalous values of the colour coefficients (TP output). These are due to atmospheric-instrumental perturbations at some epochs; so the associated frames must also be removed from the database. The final records were based on $163 \mathrm{~g}$-band and $142 r$-band useful exposures of 100-200 seconds [16]. The average magnitudes and photometric uncertainties were $m_{\mathrm{A}} \sim m_{\mathrm{B}} \sim 17.2 \mathrm{mag}$ ( $g$-band $)$ and $m_{\mathrm{A}} \sim m_{\mathrm{B}} \sim 17 \mathrm{mag}$ ( $r$-band), and $\sigma\left(m_{\mathrm{A}}\right) \sim \sigma\left(m_{\mathrm{B}}\right) \sim 16 \mathrm{mmag}$ ( $g$-band) and $\sigma\left(m_{\mathrm{A}}\right) \sim \sigma\left(m_{\mathrm{B}}\right) \sim 12 \mathrm{mmag}$ ( $r$-band). Thus, photometry to the $1 \%-2 \%$ was achieved for the lensed QSO. All these results appear in Table 2. With respect to the observed variability, we found several 100-200 mmag intrinsic events and gradients. The final light curves of the A image in the 2005-2006 and 2006-2007 seasons are displayed in Figure 3. We present both the $g$-band (green circles) and $r$-band (red triangles) curves, where the redder $(r$-band) record is shifted by +0.229

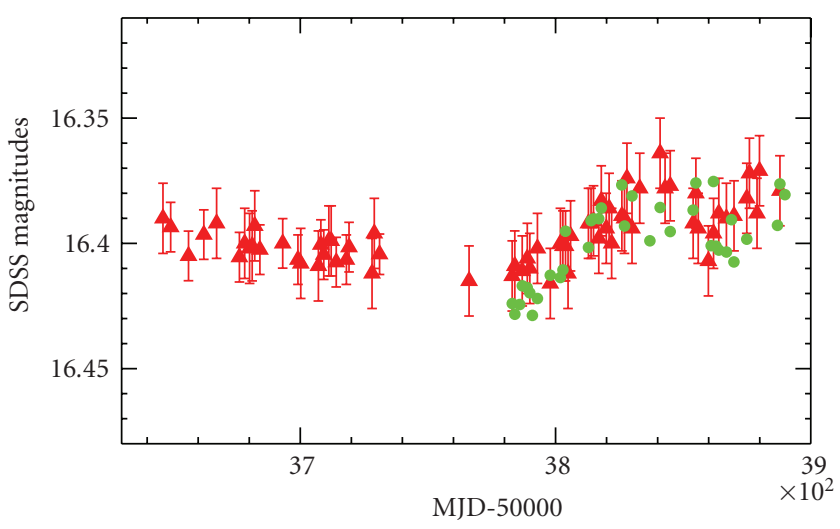

FIGURE 2: LRT light curves of SBS 0909 + 532A from October 2005 to June 2006. The whole $r$-band record (red triangles) is compared to the $g$-band curve (green circles) over the second half of the 20052006 season (it is shifted by $-0.57 \mathrm{mag}$ ).

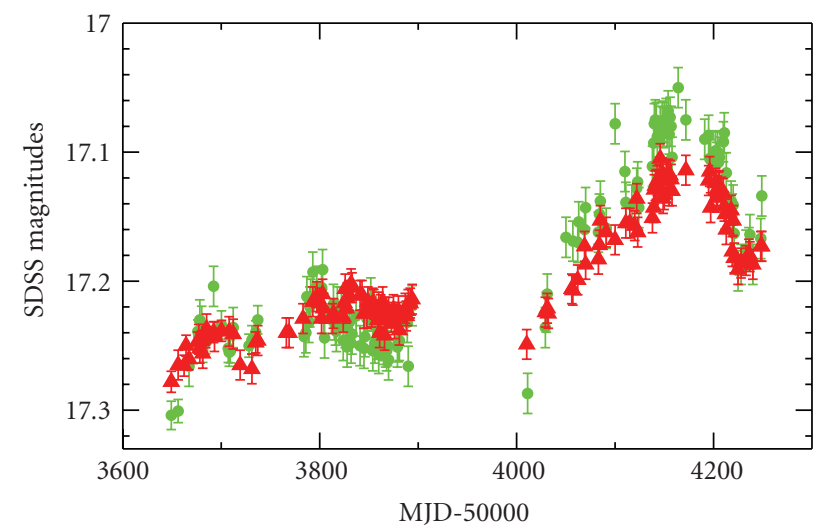

FIGURE 3: LRT light curves of QSO 0957 + 561A from October 2005 to June 2007. The $r$-band (red triangles) and $g$-band (green circles) curves are compared with each other. To make the comparison easier, the $r$-band magnitudes are shifted by +0.229 mag.

mag (average $g-r$ colour). The $r$-SDSS record from October 2005 to June 2007 (two whole seasons) incorporates different prominent features that are also seen in the $g$-SDSS curve (see Figure 3), with the $g$-SDSS features having a larger amplitude.

After running the CPP, the combined frames of FBQ $0951+2635$ in the $i$ band were selected on the basis of their image quality: FWHM $<1.5^{\prime \prime}$ and $\operatorname{SNR}(\mathrm{S} 3)>50$, where the S3 field star has a magnitude similar to that of the faintest QSO image B $\left(m_{\mathrm{A}} \sim 17.5 \mathrm{mag}\right.$ and $\left.m_{\mathrm{B}} \sim 18.6 \mathrm{mag}\right)$. Therefore, the TP was exclusively applied to 22 combined exposures of mostly 400-500 seconds [19]. Unfortunately, we could not achieve 1\%-2\% level photometry for B even using frames with $\langle\mathrm{FWHM}\rangle \sim 1.2^{\prime \prime}$ and $\langle\mathrm{SNR}(\mathrm{S} 3)\rangle \sim 80$. Some deviations between fluxes of adjacent nights were very large (see green triangles in Figure 4), and we did not try to improve the B record (subtracting galaxy contaminations, looking for outliers, grouping adjacent fluxes, etc). The light curve of A is much less noisy, and so an accurate trend could be obtained by grouping four pairs of adjacent fluxes 


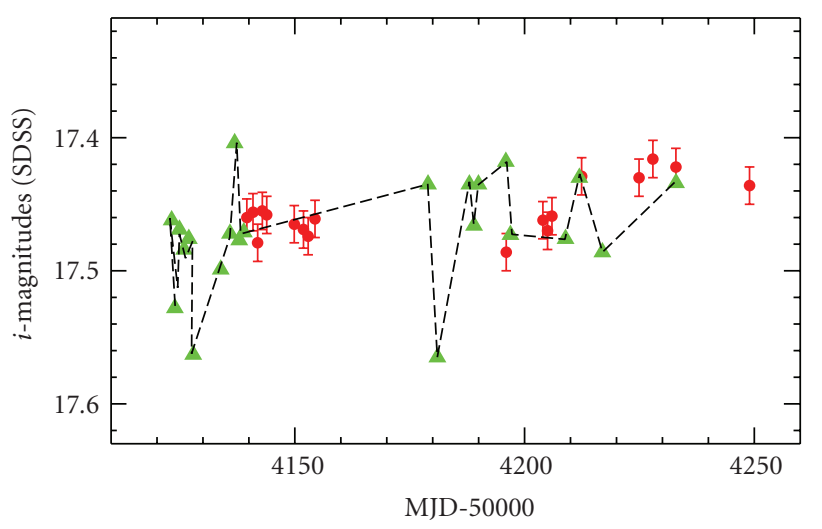

FIgURE 4: LRT $i$-band light curves of FBQ $0951+2635$ in FebruaryMay 2007. We compare the record of the A image (red circles) and the time- and magnitude-shifted light curve of the B image (green triangles; the noisy brightness record of B is shifted in time and magnitude, using the 16-day time delay of the system and an average magnitude difference of $1.094 \mathrm{mag}$ ). There is a reasonable agreement between both trends, and so we do not find evidence of short-timescale extrinsic variability.

(showing significant differences between pair members). The final record of A (18 data points; see red circles in Figure 4) is characterised by a typical uncertainty $\sigma\left(m_{\mathrm{A}}\right) \sim 14 \mathrm{mmag}$, and it shows a 60 mmag gradient.

As a general conclusion, the LRT works reasonably well when monitoring the 17-mag $r$-band images of two of the brightest double QSOs. Nightly 120 seconds exposures permit us to get accurate and reliable fluxes, approximately 2 times per week. Although this sampling efficiency is partially due to weather, technical factors play also a role (see Section 6). While the fluxes of the images of the wideseparation system $\left(\Delta \theta_{\mathrm{AB}} \gg 1^{\prime \prime}\right)$ have $12 \mathrm{mmag}$ errors $(\sim 1 \%$ accuracy in physical flux), the study of the compact system leads to larger uncertainties ( $2 \%$ accuracy in physical flux). In the current (second) phase of the LQLM project, we are trying to characterise the performance of the LRT when monitoring fainter (18-19 mag) $r$-band images of double and quadruple QSOs from nightly exposures of 300-600 seconds (see Section 6).

\section{Main Scientific Outputs}

5.1. Time Delays. The accurate $r$-band light curves of SBS $0909+532$ allowed us to confirm the time delay of the system (about 50 days) [7]. On the other hand, the QSO $0957+561$ records led to accurate time delays between QSO images and optical bands [16]. These new delays (together with previous ones obtained by the Princeton group and collaborators [20]) suggested the existence of reverberation in the accretion disc around the central black hole (BH; see Figure 5). High-energy (EUV/X-ray) flares are generated within or close to the jet and later reprocessed by the disc in two different emission rings (blue and red rings in Figure 5, corresponding to the $g$ and $r$ observation bands, resp.). The fluctuations at shorter wavelengths (inner ring) are then

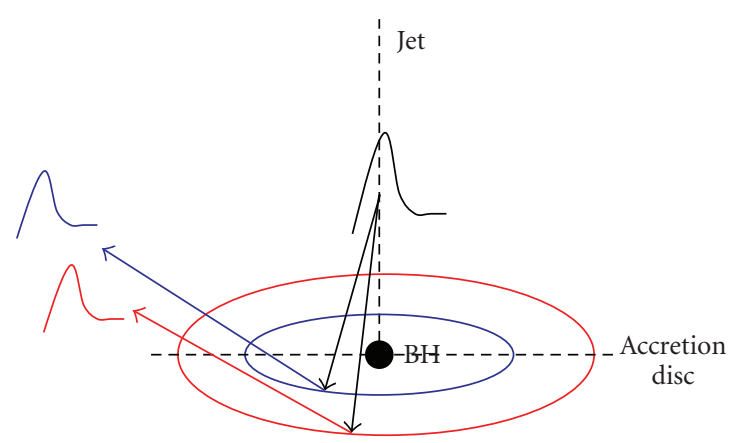

FIGURE 5: Reverberation in the accretion disc around the central BH of QSO $0957+561$.

produced and observed before than those similar variations at larger wavelengths (outer ring).

5.2. Structure Functions. The brightness records of SBS 0909 +532 ( $r$ band) and QSO $0957+561$ ( $g$ and $r$ bands) are important tools to better understand the mechanism of intrinsic variability in QSOs at $1<z<2$. Both double QSOs have similar redshift $(z \sim 1.4)$, BH mass $\left(M_{\mathrm{BH}} \sim\right.$ $2-4 \times 10^{9} \mathrm{M}_{\odot}$ ), and luminosity [21]; so similar fluctuations might be produced in both distant sources. In the first study of the structure function of SBS $0909+532$, no simple model was able to accurately reproduce the typical luminosity fluctuations at rest-frame lags shorter than 100 days [7]. However, if we compute more realistic uncertainties in the structure function, that is, considering that not all pairs of data in a given time lag bin are independent [22], 100day time-symmetric flares can account for the fluctuations at $\lambda_{\text {rest }} \sim 2600 \AA$ (reduced $\left.\chi^{2} \sim 1\right)$.

We also obtained the structure functions of the QSO $0957+561$ luminosity at $\lambda_{\text {rest }} \sim 2100 \AA$ ( $g$ band) and $2600 \AA ̊$ ( $r$ band) [23]. In Figure 6, we show the three LQLM I structure functions. For QSO $0957+561$, we did not find clear evidence of a chromatic mechanism of variability, since the fluctuations at the two rest-frame wavelengths were explained from the production of 100-day time-symmetric and 170-day time-asymmetric flares. A time-asymmetric flare refers to a shot with very different rise and decay times, whereas a time-symmetric flare has symmetric rise and decay. Although there are subtle differences between the two structure functions of QSO $0957+561$ (see Figure 6), these are likely caused by the difference between time coverages, gaps, and artifacts in the $g\left(\lambda_{\text {rest }} \sim 2100 \AA\right)$ and $r\left(\lambda_{\text {rest }} \sim\right.$ $2600 \AA$ Á) bands [23]. Besides of the time delay measurements, our structure function analysis also supports a reverberation scenario (see Figure 5). As time-symmetric flares lasting $\sim 100$ days are also generated in SBS $0909+532$ (see above), this fact suggests a unified scheme. In other words, the SBS $0909+532$ flares at $\lambda_{\text {rest }} \sim 2600 \AA$ may be due to high-energy symmetric fluctuations similar to those occurring in QSO $0957+561$. High-energy asymmetric fluctuations (lasting $\sim 170$ days) would be produced in an intermittent way, since they are not detected in our SBS $0909+532$ records and 


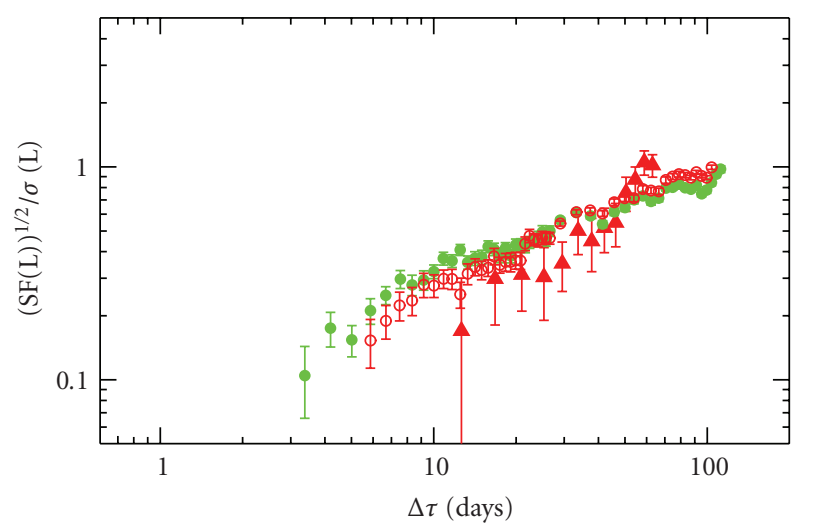

- SBS $0909+532(2600 \AA)$

- QSO $0957+561(2100 \AA)$

- QSO $0957+561(2600 \AA)$

FIGURE 6: LQLM I structure functions of the UV variability of two lensed QSOs.

previous records of QSO $0957+561$ (that were obtained 10 years before).

The on-going monitoring of QSO $0957+561$ will permit us to very accurately trace the structure at $\Delta \tau \leq 100$ days as well as to properly address the structure at rest-frame lags exceeding $\Delta \tau \sim 100$ days. In a forthcoming analysis, from a longer monitoring period, we will describe the UV variability at rest-frame lags $\leq 1$ year.

5.3. Extrinsic Variations. We have not detected any kind of extrinsic signal. If the light curves of SBS $0909+532$, QSO $0957+561$, and FBQ $0951+2635$ incorporate extrinsic variations, these should be very weak (undetectable in our experiments). Thus, we indeed put constraints on the possible extrinsic signals. These constraints can be used to obtain information on the composition of the lensing halos and the structure of the distant sources (see Introduction and $[7,16,24])$.

\section{Archive and LQLM II Subproject}

All LQLM I optical frames are publicly available at the Liverpool Quasar Lens Monitoring archive [25] on the German Astrophysical Virtual Observatory (GAVO). This archive is part of a larger optical database of gravitationally lensed QSOs. The whole collection of frames consists of exposures from three telescopes in the North Hemisphere: $3.5 \mathrm{~m}$ ARC Telescope at the Apache Point Observatory (USA), $2.0 \mathrm{~m}$ LRT at the Roque de los Muchachos Observatory (Canary Islands, Spain), and $1.5 \mathrm{~m} \mathrm{AZT-22} \mathrm{at} \mathrm{the} \mathrm{Maidanak} \mathrm{Observatory}$ (Uzbekistan). We point out that much of these frames at the global Lens Image Archive on the GAVO are not in the public domain, but the person-in-charge of the Maidanak database could make them available upon request.

In the LQLM public archive, for example, you may select a target and a filter and then get details on observation dates, labels of FITS files (frames), exposure times (in s),

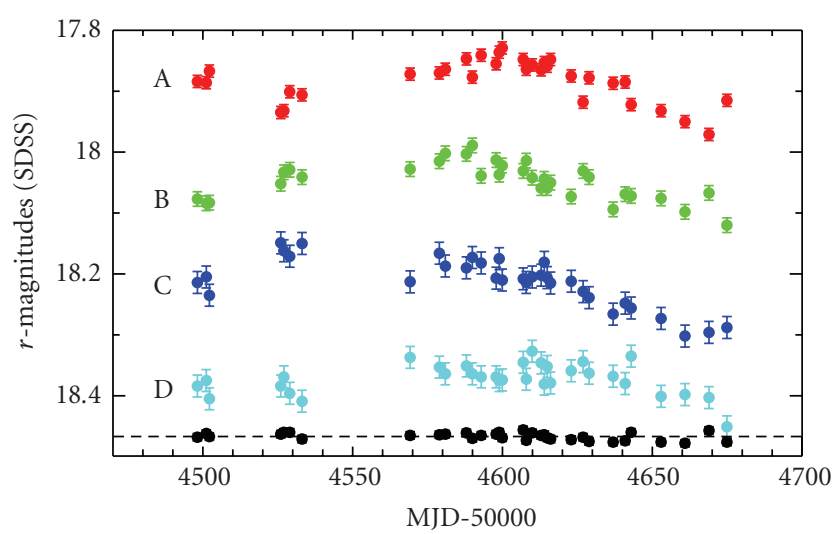

FIgURE 7: LRT light curves of the four images A-D of $\mathrm{H} 1413+117$ (Cloverleaf QSO). These $r$-band records correspond to the LQLM II observational subprogram.

file sizes, air masses, Moon illuminations (fractions), lunar distances (in deg), backgrounds (in counts $\mathrm{s}^{-1}$ ), and seeing values (in "). We also manage a website incorporating information on the status of the LQLM project. In particular, the LQLM I data-tools release [26] includes a link to the archive as well as public access to some photometric pipelines and their outputs (quality of images, instrumental fluxes, transformation coefficients, calibrated and corrected magnitudes, etc.). The data-tools release also contains final light curves and references.

The second observational phase of the LQLM project started in February 2008, and it will be completed in the middle of 2010 or early 2011. This LQLM II observational subprogram is designed to take frames for a broad range of lensed QSOs. The main targets are the very bright doubles observed during the first phase as well as other bright double and quadruple QSOs. For example, the Cloverleaf QSO (H $1413+117$ ) consists of four images A-D of a QSO at $z=$ 2.55 [12]. A photometric model with four close stellar-like sources, that is, four empirical PSFs, is able to fully describe the compact lens system. From this photometric model, we infer the light curves in Figure 7. The LQLM II records in Figure 7 are based on 300 seconds $r$-band exposures taken over a semester (February-July 2008). Apart from the curves of the four quasar images (A-D), the light curve of a control (non-variable) star is also plotted for comparison (black circles and dashed horizontal line; the stellar brightness is shifted by $+0.3 \mathrm{mag}$ ). We remark that the QSO records from our best 33 frames, that is, from a set of exposures having $\langle\mathrm{FWHM}\rangle \sim 1.2^{\prime \prime}$ and $\langle\mathrm{SNR}(\mathrm{star})\rangle \sim 200$, are characterised by small observational noises: $\sigma\left(m_{\mathrm{A}}\right) \sim 10 \mathrm{mmag}\left(m_{\mathrm{A}} \sim\right.$ $17.9 \mathrm{mag}), \sigma\left(m_{\mathrm{B}}\right) \sim 12 \mathrm{mmag}\left(m_{\mathrm{B}} \sim 18 \mathrm{mag}\right), \sigma\left(m_{\mathrm{C}}\right) \sim$ $18 \mathrm{mmag}\left(m_{\mathrm{C}} \sim 18.2 \mathrm{mag}\right)$, and $\sigma\left(m_{\mathrm{D}}\right) \sim 18 \mathrm{mmag}\left(m_{\mathrm{D}} \sim\right.$ $18.4 \mathrm{mag})$.

The LRT performance has improved during the last observing semesters (LQLM II period), and this is a promising result to make a high-quality $r$-band database (QSO images with $m_{r} \leq 19 \mathrm{mag}$ ) leading to $1 \%-2 \%$ accuracy and reasonable sampling. Excluding two relatively long gaps in the light curves of H $1413+117$ (see Figure 7), the effective 
sampling period is about 4 days. For the very bright and widely separated double QSO $(0957+561)$, we have obtained an effective sampling period of about 2.4 days over the first semester of 2008 and the 2008-2009 season (from November 2008 to June 2009). This represents a sampling efficiency significantly better than the previous one (see Table 2 ).

\section{Acknowledgments}

The authors are grateful to the organizers (LOC and SOC) of the workshop on Robotic Autonomous Observatories held in Malaga from 18 to 21 May 2009, who prepared a useful meeting showing current robotic facilities as well as the efforts of several groups in different fields and some ongoing projects. They thank several members of the Liverpool Telescope staff (M. Bode, C. Moss, R. Smith, and I. Steele) for guidance in the preparation of their robotic monitoring project and kind interaction during the last five years. The Liverpool Telescope is operated on the island of La Palma by Liverpool John Moores University in the Spanish Observatorio del Roque de los Muchachos of the Instituto de Astrofisica de Canarias with financial support from the UK Science and Technology Facilities Council. They thank R. Gil-Merino and B. McLeod for different kinds of support during the first stages of the LQLM project. Their LQLM project is being developed in parallel with a similar project at the Maidanak Observatory. They also thank T. Akhunov, B. Artamonov, O. Burkhonov, E. Koptelova, S. Nuritdinov, A. Sergeyev, and A. Zheleznyak for collaboration, discussion and exchange of information on common targets. They use information taken from the Sloan Digital Sky Survey (SDSS) Web site, and they are grateful to the SDSS team for doing that public database. This research has been financially supported by the Spanish Department of Education and Science Grants AYA2004-08243-C03-02 and AYA2007-67342-C03-02, and University of Cantabria funds.

\section{References}

[1] P. Schneider, J. Ehlers, and E. E. Falco, Gravitational Lenses, Springer, Berlin, Germany, 1992.

[2] S. Refsdal, "On the possibility of determining Hubble's parameter and the masses of galaxies from the gravitational lens effect," Monthly Notices of the Royal Astronomical Society, vol. 128, pp. 307-310, 1964.

[3] C. S. Kochanek, P. Schneider, and J. Wambsganss, Gravitational Lensing: Strong, Weak \& Micro, Springer, Berlin, Germany, 2006.

[4] A. Yonehara, "Source size limitation from variabilities of a lensed quasar," Astrophysical Journal, vol. 519, no. 1, pp. L31L34, 1999.

[5] L. J. Goicoechea, "Multiple delays in QSO 0957+561: observational evidence and interpretation," Monthly Notices of the Royal Astronomical Society, vol. 334, no. 4, pp. 905-911, 2002.

[6] T. Kawaguchi, S. Mineshige, M. Umemura, and E. L. Turner, "Optical variability in active galactic nuclei: starbursts or disk instabilities?" The Astrophysical Journal, vol. 504, no. 2, pp. 671-679, 1998.

[7] L. J. Goicoechea, V. N. Shalyapin, E. Koptelova, R. Gil-Merino, A. P. Zheleznyak, and A. Ullán, "First robotic monitoring of a lensed quasar: intrinsic variability of SBS 0909+532," New Astronomy, vol. 13, no. 3, pp. 182-193, 2008.

[8] D. Parafiez, J. Hjorth, I. Burud, P. Jakobsson, and Á. Elíasdóttir, "Microlensing variability in time-delay quasars," Astronomy and Astrophysics, vol. 455, no. 1, pp. L1-L4, 2006.

[9] J. Wambsganss, Gravitational microlensing, Ph.D. thesis, Munich University, Munich, Germany, 1990.

[10] C. S. Kochanek, "Quantitative interpretation of quasar microlensing light curves," The Astrophysical Journal, vol. 605, no. 1, pp. 58-77, 2004.

[11] R. Gil-Merino, J. González-Cadelo, L. J. Goicoechea, V. N. Shalyapin, and G. F. Lewis, "Is there a caustic crossing in the lensed quasar Q2237+0305 observational data record?" Monthly Notices of the Royal Astronomical Society, vol. 371, no. 3, pp. 1478-1482, 2006.

[12] C. S. Kochanek, E. E. Falco, C. Impey, et al., "Gravitational Lens Data Base," 2009, http://www.cfa.harvard.edu/castles/.

[13] I. A. Steele, “The Liverpool robotic telescope," New Astronomy Reviews, vol. 45, no. 1-2, pp. 45-47, 2001.

[14] I. A. Steele, R. J. Smith, P. C. Rees, et al., "The Liverpool telescope: performance and first results," in Ground-based Telescopes, vol. 5489 of Proceedings of SPIE, pp. 679-692, Glasgow, UK, June 2004.

[15] B. A. McLeod, G. M. Bernstein, M. J. Rieke, and D. W. Weedman, "The gravitational lens MG 0414+0534: a link between red galaxies and dust," The Astronomical Journal, vol. 115, no. 4, pp. 1377-1382, 1998.

[16] V. N. Shalyapin, L. J. Goicoechea, E. Koptelova, A. Ullán, and R. Gil-Merino, "New two-colour light curves of Q0957+561: time delays and the origin of intrinsic variations," Astronomy and Astrophysics, vol. 492, no. 2, pp. 401-410, 2008.

[17] J. Manfroid, F. Selman, and H. Jones, "Achieving 1\% photometric accuracy with the ESO Wide Field Imager," The Messenger, vol. 104, pp. 16-20, 2001.

[18] E. A. Magnier and J.-C. Cuillandre, "The Elixir system: data characterization and calibration at the Canada-France-Hawaii Telescope," The Publications of the Astronomical Society of the Pacific, vol. 116, no. 819, pp. 449-464, 2004.

[19] V. N. Shalyapin, L. J. Goicoechea, E. Koptelova, et al., "Microlensing variability in FBQ 0951+2635: short-timescale events or a long-timescale fluctuation?" Monthly Notices of the Royal Astronomical Society, vol. 397, pp. 1982-1989, 2009.

[20] T. Kundić, E. L. Turner, W. N. Colley, et al., "A robust determination of the time delay in $0957+561 \mathrm{~A}, \mathrm{~B}$ and a measurement of the global value of Hubble's constant," The Astrophysical Journal, vol. 482, no. 1, pp. 75-82, 1997.

[21] C. Y. Peng, C. D. Impey, H.-W. Rix, et al., "Probing the coevolution of supermassive black holes and galaxies using gravitationally lensed quasar hosts," The Astrophysical Journal, vol. 649, no. 2, pp. 616-634, 2006.

[22] S. Collier and B. M. Peterson, "Characteristic ultraviolet/optical timescales in active galactic nuclei," The Astrophysical Journal, vol. 555, no. 2, pp. 775-785, 2001.

[23] L. J. Goicoechea, V. N. Shalyapin, R. Gil-Merino, and A. Ullán, "Structure function of the UV variability of Q0957+561," Astronomy and Astrophysics, vol. 492, no. 2, pp. 411-417, 2008.

[24] R. Schmidt and J. Wambsganss, "Limits on MACHOs from microlensing in the double quasar Q0957+561," Astronomy and Astrophysics, vol. 335, no. 1, pp. 379-387, 1998.

[25] http://dc.zah.uni-heidelberg.de/liverpool/res/rawframes/q/ form.

[26] http://grupos.unican.es/glendama/LQLMI_DR.htm. 

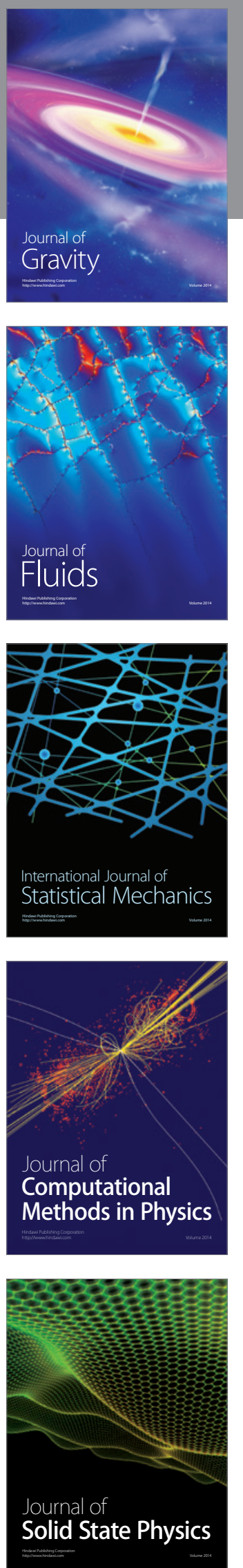

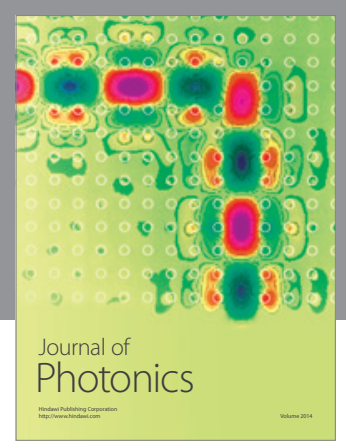

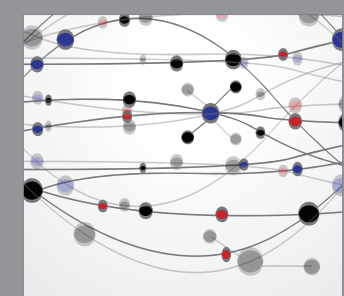

The Scientific World Journal
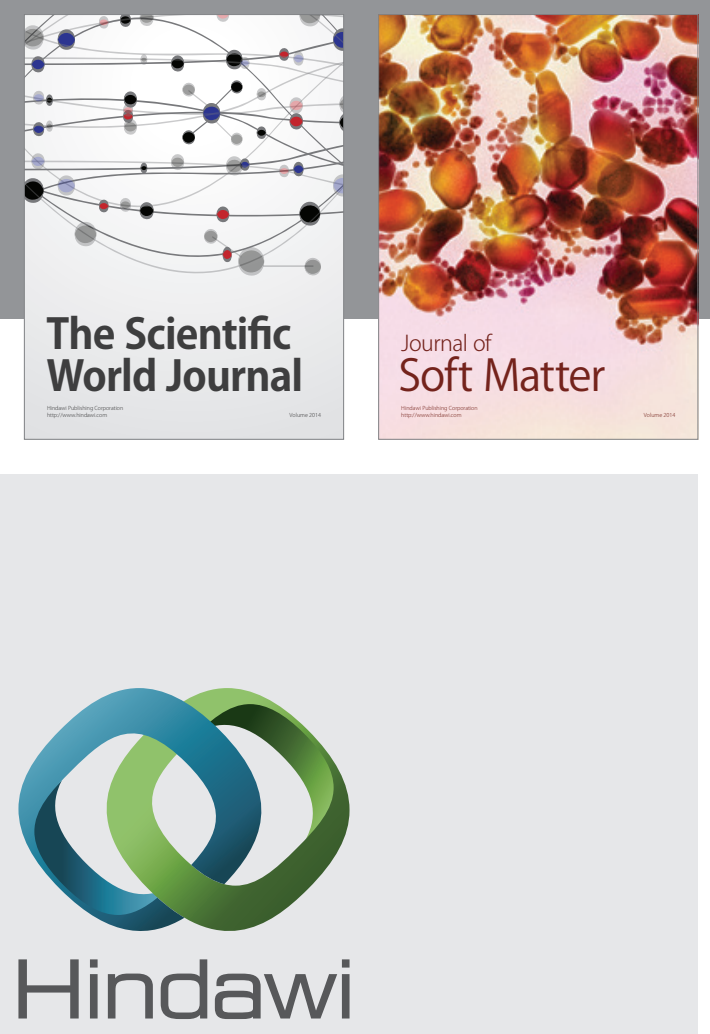

Submit your manuscripts at

http://www.hindawi.com
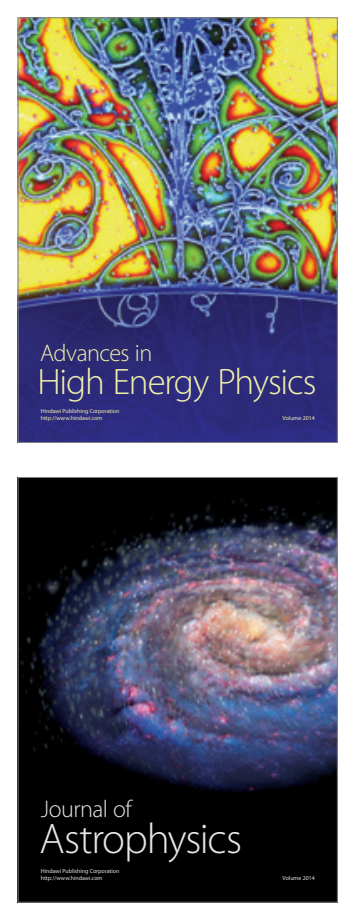
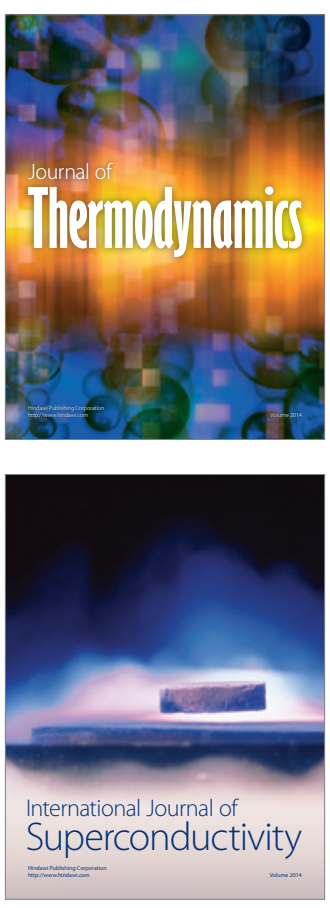
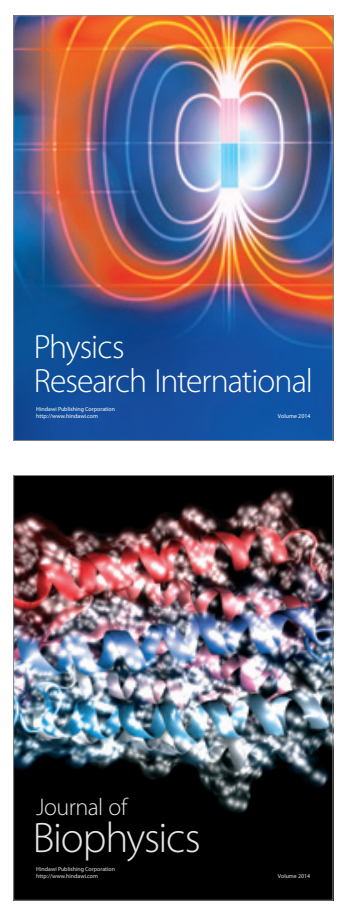
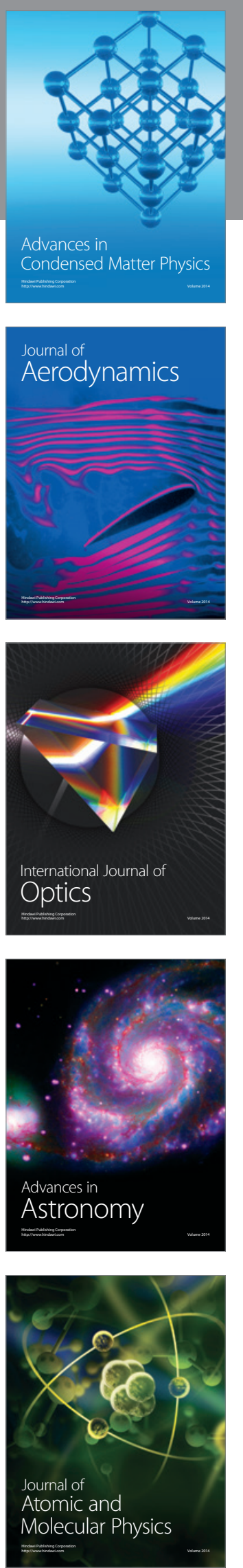